\title{
Scale invariance and universality of force networks in static granular matter
}

\author{
Srdjan Ostojic ${ }^{1}$, Ellák Somfai $^{2}$ \& Bernard Nienhuis ${ }^{1}$ \\ ${ }^{1}$ Institute for Theoretical Physics, Universiteit van Amsterdam, Valckenierstraat 65, 1018 XE Am- \\ sterdam, Netherlands \\ ${ }^{2}$ Universiteit Leiden, Instituut-Lorentz, PO Box 9506, 2300 RA Leiden, Netherlands
}

Force networks form the skeleton of static granular matter ${ }^{12}$. They are the key ingredient to mechanical properties, such as stability ${ }^{3}$, elasticity ${ }^{45}$ and sound transmission ${ }^{67}$, which are of utmost importance for civil engineering and industrial processing. Previous studies have focused on the global structure of external forces 8910 (the boundary condition), and on the probability distribution of individual contact forces ${ }^{412}$. The disordered spatial structure of the force network, however, has remained elusive so far. Here we report evidence for scale invariance of clusters of particles that interact via relatively strong forces. We analyzed granular packings generated by molecular dynamics simulations mimicking real granular matter; despite the visual variation, force networks for various values of the confining pressure and other parameters have identical scaling exponents and scaling function, and thus determine a universality class. Remarkably, the flat ensemble of force configurations 131415 _ a simple generalization of equilibrium statistical mechanics-belongs to the same universality class, while some widely studied simplified models 1617 do not.

Sand dunes, piles of apples in a supermarket, and coffee beans in a jar are all examples of 
static granular matter. In these examples the particles (sand grains, apples, coffee beans) interact with their neighbors with repulsive contact forces forming a network. Due to disorder (even in a regular pile each apple is slightly different) the force network is inhomogeneous in space 1920 . The simplest characterization of the force network is the probability distribution of individual contact forces 2122 23: $P(F)$. In typical granular packings $P(F)$ has an exponential tail at large $F$, and a plateau at small $F$. While $P(F)$ is an important and well studied quantity, it tells nothing about the spatial structure of the force network, which is so striking visually, as can be seen on Fig. 11 a. As a large contact force on one side of a particle is typically balanced by another large force on the opposite side, large forces tend to align in filamentary structures, called force chains though they are not completely linear. The force chains are stabilized by weaker side contacts, and form a disordered branching network resembling a fractal. Typical fractals are invariant under a change of length scale. A quantitative analysis of that scale-invariance is however difficult, in part because there is no sharp distinction between the force chains and the background contacts.

In this Letter we show that discriminating between forces of different magnitudes provides a quantitative characterization of force chains. Via an analogy with equilibrium critical phenomena, such a description naturally captures scale invariance: a set of scaling exponents (fractal dimensions) and a scaling function are extracted from a given ensemble of force networks. We applied this approach to two-dimensional granular packings under isotropic pressure generated by molecular dynamics simulations. Although the appearance of the force network varies significantly with pressure and polydispersity, we found that the scaling exponents and the scaling function are independent of these and other parameters, and thus capture universal properties of force networks. 
We also show that a theoretical model based on the extension of equilibrium statistical mechanics proposed by Edwards faithfully represents this universality class, while some other simple models do not.

The force network in a granular packing can be represented as a set of bonds connecting grains in contact. Each bond carries a scalar given by the magnitude of the repulsive force between the grains (see Fig. 11). To quantify the, visually obvious, force chains in such a network, we choose a threshold $f$, and consider only chains formed by bonds carrying forces larger than $f$. Rather than select a fixed arbitrary value of $f$, we study such structures at different scales by varying the threshold. For small values, most of the grains remain connected, but as the threshold is increased the packing breaks up into disconnected clusters (see Fig. 2). The extent of each force chain can then be characterized by the size of the corresponding cluster, i.e. the number of mutually connected bonds.

The force network varies totally between packings created under the same external conditions, so that a statistical approach is needed. Following the above procedure, force chains in an ensemble of packings can be described by the probability $P(s, f)$ that, at a threshold $f$, a given random grain belongs to a cluster of size $s$. Similar statistical descriptions in terms of clusters are common in the theory of equilibrium phase transitions 24 , where the threshold $f$ plays the role of temperature. As in a percolation model $\frac{25}{\text {, }}$, a phase transition occurs at the critical threshold $f_{c}$ above which no cluster connects the opposite boundaries of the system. Around this value, the system exhibits scale invariance, in particular the probability distribution of cluster sizes can be 

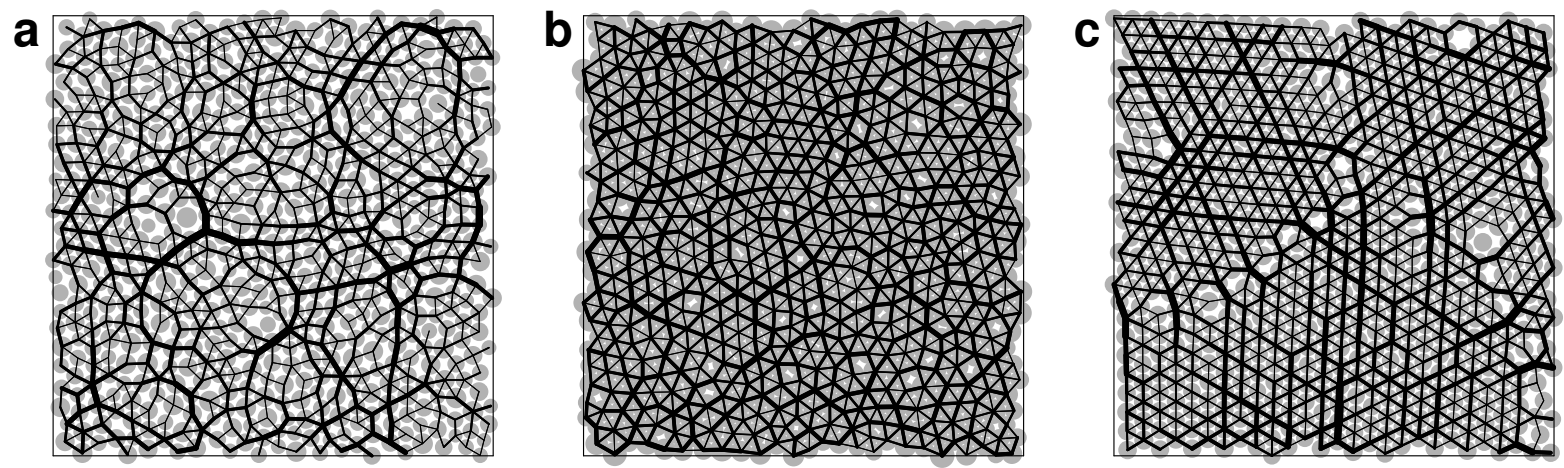

Figure 1: Dependence of the force network on pressure and polydispersity. Grains are represented as gray disks and forces as bonds. The thickness of each bond is proportional to the magnitude of the force. Packing under (a) pressure $p=10^{-4}$ (in rescaled units) and polydispersity $d=20 \%$ (b) $p=10^{-1}$ and $d=20 \%$, (c) $p=10^{-2}$ and $d=5 \%$

expressed as $P(s, f) \sim s^{-\tau} \rho\left(s /\left(f-f_{c}\right)^{\sigma}\right)$. This scale invariance is universal: the scaling exponents $\tau$ and $\sigma$, as well as the scaling function $\rho$ are determined only by the global symmetries of the system rather than the microscopic details of the interactions. Equilibrium critical phenomena can therefore be classified in a discrete number of universality classes according to the values of the exponents.

Such an analogy with equilibrium critical phenomena suggests the existence of scale invariance around the threshold $f_{c}$ below which one of the clusters spans the entire packing. The associated scaling exponents and scaling function would provide a novel characterization of the spatial organization of forces in granular matter. To study the existence of scale invariance and universality, we examine the behavior of the $n^{\text {th }}$ moment $m_{n}$ of $P(s, f)$ as function of the threshold $f$ and the system size $N$ (number of contacts in the packing). We look for scaling as function 

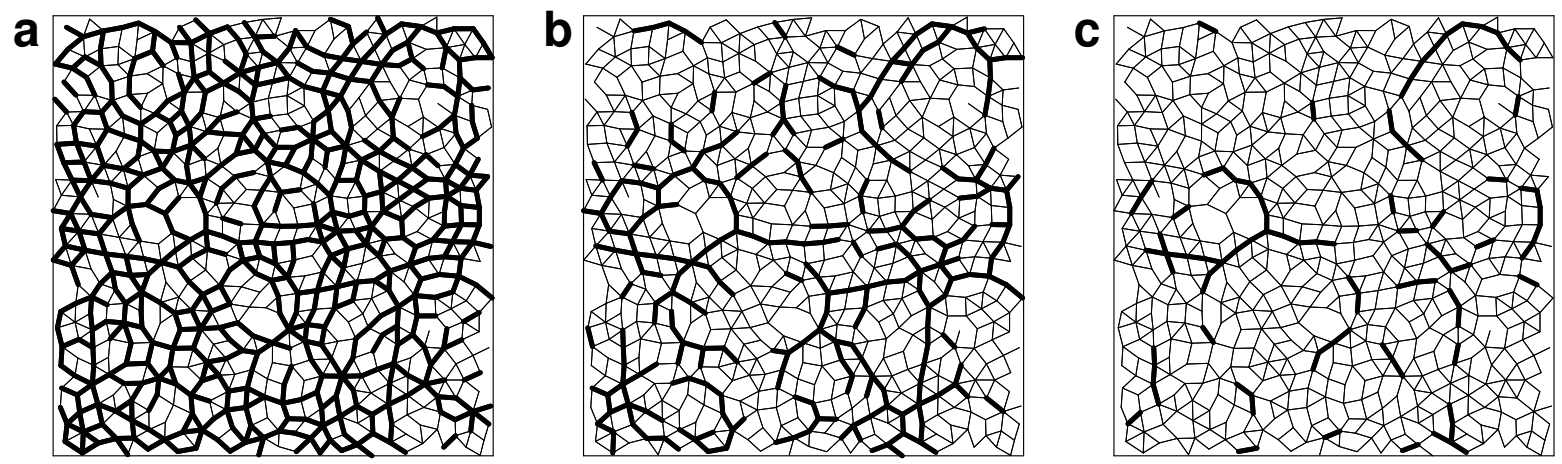

Figure 2: Force chains at different scales. The packing of Fig. 1 a is repeated here, showing as bold lines forces larger then a threshold $f$. The threshold values are (a) $f=0.5 f_{c}$, (b) $f=f_{c}$ and (c) $f=1.5 f_{c}$, where $f_{c}$ is the critical threshold.

of the system size, which in case of equilibrium critical phenomena takes the form

$$
m_{n}(f, N) \sim N^{\phi_{n}} M_{n}\left(\left[f-f_{c}\right] N^{1 / 2 \nu}\right)
$$

where the scaling function $M_{n}$ is related via integration to $\rho$, and the scaling exponents are given by $\phi_{n}=(n+1-\tau) /(\tau-1)$ and $\nu=(\tau-1) / 2 \sigma$. Here we present a study for $n=2$ because it is the lowest moment to diverge, but the results hold also for higher moments.

We have carried out a scaling analysis on packings created by molecular dynamics (MD) simulations (also called discrete elements methods) of a system of polydisperse spheres in a periodic two-dimensional cell subject to an isotropic pressure. The particles are deformable and interact via nonlinear Hertz-Mindlin forces (see Methods: Molecular Dynamics). We first consider strongly polydisperse frictionless packings at low pressure, leading to strongly disordered force networks with clearly visible force chains shown in Fig. 11a. For different system sizes, we determined the force-chain clusters at a large number of threshold values and thus computed $m_{2}(f, N)$, the second 
moment of clusters sizes (leaving out the largest cluster in each sample). If $m_{2}$ scales according to Eq. (1), then plotting $N^{-\phi} m_{2}$ as function of $\left(f-f_{c}\right) N^{1 / 2 \nu}$ should lead to a collapse of data for different system sizes on a single curve. Varying $\phi, \nu$ and $f_{c}$, a good collapse is indeed obtained for $\phi=0.89 \pm 0.01$ and $\nu=1.6 \pm 0.1$, clearly confirming scale invariance.

In order to test the dependence on various parameters, we repeated the procedure for packings created at different pressures, polydispersities, coefficients of friction and force-laws. As the pressure is increased, the grains deform and the number of contacts per particle rises (see Fig. 2 a). The force network becomes increasingly uniform in appearance, and the distribution of force magnitudes narrower (see Supplementary Information). Computing $m_{2}(f)$ for values of pressure spanning three orders of magnitude, we nevertheless find that the optimal data collapse is obtained in the same range $\phi=0.89 \pm 0.01$ and $\nu=1.6 \pm 0.1$ independently of the pressure. Decreasing the polydispersity leads to crystallization of the grains (see Fig. 2]b), however such ordering of the contact network also leaves the values of the scaling exponents unchanged. Moreover, although friction leads to smaller coordination number in the packing, it does not influence the scaling properties. In this case the forces are not normal to the grains, but tangential forces are typically much smaller then $f_{c}$ (see Supplementary Information), so that they do not affect the scaling of cluster sizes. Finally, particles interacting with harmonic forces instead of non-linear Hertz forces lead once again to the same exponents.

The independence of the exponents on pressure, polydispersity, friction and force law is clear evidence of universality. On the other hand, as expected the value of the critical threshold is not 
universal, it varies between 1.3 and 1.6 times the average normal force, and depends on all the parameters. Moreover the width and height of the scaling function vary with the parameters: the width decreases with increasing pressure, while the height increases with increasing polydispersity. A linear rescaling of both axes nevertheless leads to a single collapse for all considered values of parameters, displayed in Fig. 3. similarly to equilibrium critical phenomena, the full scaling function appears to be universal.

As the scaling exponents and the scaling function are universal, they can be obtained from simplified models in the appropriate universality class. Years ago, Edwards proposed to generalize the micro canonical principle of thermodynamics to jammed systems such as granular matter 13 . The key idea is to ignore history and treat as equally likely all stable configurations of grains. The

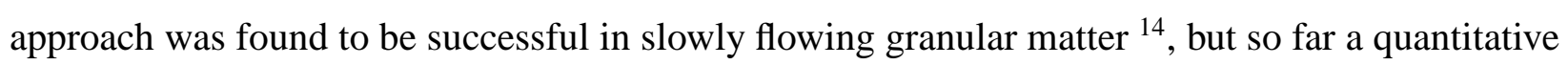
confirmation in the case of static granular matter has been lacking.

A simple extension of this micro-canonical ensemble to a packing of grains in a fixed geometry was named the "snooker packing" 15. It consists of a hexagonal arrangement of rigid, frictionless and mono-disperse spheres confined within a triangular domain, with the same pressure applied to all sides of the packing. In such a system, force balance alone does not completely determine the forces. Here it is natural to generalize Edwards' approach by treating as equally likely all arrangements of repulsive forces in balance on each grain, while keeping fixed the geometry of contacts. Using Monte Carlo simulations (see Methods: Monte Carlo), we generated force networks on the snooker packing with uniform probability, and examined the scaling of the second 


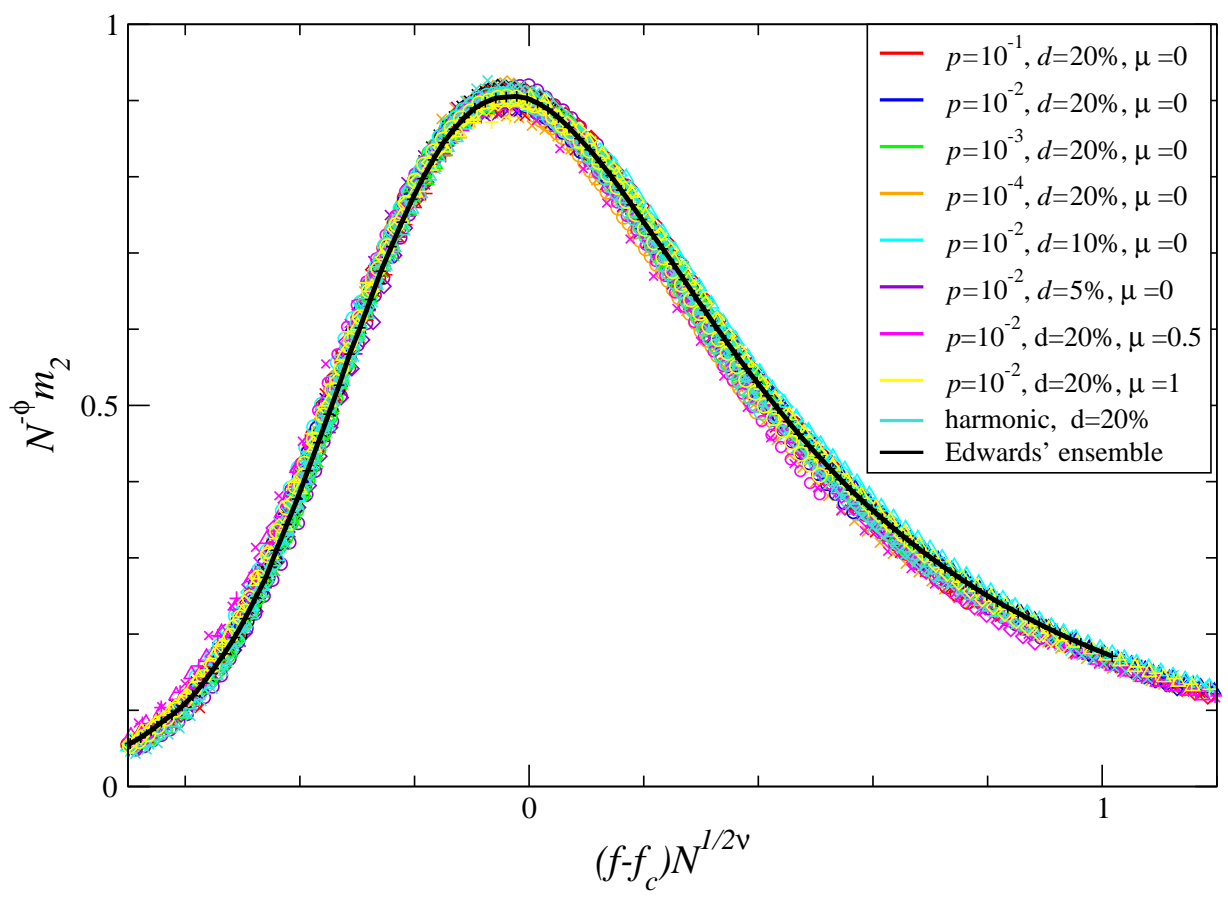

Figure 3: The universal scaling function. The mean square of the cluster sizes, omitting the largest cluster, $m_{2}$, is rescaled as $B N^{-\phi} m_{2}$ and plotted as function of the rescaled threshold $A\left(f-f_{c}\right) N^{1 / 2 \nu}$. The figure shows the collapse on a single curve of 50 datasets corresponding to various packing parameters and system sizes. A color is associated with each of six different sets of parameters (pressure $p$, polydispersity $d$ ) used in MD simulations. For each set of parameters, five system sizes (increasing by factors of two) are represented by symbols $\circ, \diamond, \triangle, \times,+$. The collapse was obtained for $\phi=0.89 \pm 0.1$ and $\nu=1.6 \pm 0.1$ for all datasets, while the value of $f_{c}, A$ and $B$ depends on the parameters (but not on the system size). In black we show Monte Carlo results of the Edwards' ensemble on the "snooker packing", which gives the same values of $\phi$ and $\nu$. 
moment of cluster size distribution for different system sizes. The optimal collapse is obtained for values of scaling exponents within error bars equal to those found from MD simulations. Moreover, the full scaling function appears to be identical to the one found in MD simulations as shown in Fig. 3 ,

Some other simple models of granular matter lead to different scaling exponents. For example, the extensively studied $q$-model $\frac{161718}{18}$, which implements balance between vertical forces only, leads to $\phi=0.69 \pm 0.01$ and $\nu=3.1 \pm 0.1$. Moreover, the universality class depends on whether a top-to-bottom propagation of forces is assumed (S.O and B.N., manuscript in preparation). The agreement between the Edwards ensemble and the results of molecular dynamics simulations is thus rather remarkable. In particular it shows that the elasticity of the grains and the details of the force it generates are irrelevant for the shape of the scaling function. More surprisingly, the geometrical randomness of the grain arrangements does not have any bearing on the universal mechanical properties. We expect that the key ingredients are the vectorial balance of forces on each grain, as well as the isotropy of the confining pressure: static packings under shear may lead to different scaling exponents. We conjecture that in three dimensions the exponents and scaling function are equally universal, though different from their two-dimensional equivalent.

Our results establish an unexpected connection between static granular matter and equilibrium critical phenomena. The observed scale invariance is in all aspects comparable to equilibrium criticality, and defines a novel universality class of isotropic force networks. This universality class is for example distinct from that of percolation, where the same analysis can be applied. This result 
implies the existence of long-range correlations between forces, a characteristic of structures such as force chains. We expect force networks in other jammed systems, such as foams and emulsions, to belong to the same universality class. We hope that recent progress in the measurement of inter-grain and inter-bubble forces 1226 will lead to an experimental evaluation of the scaling exponents.

\section{Methods}

Molecular dynamics. We use molecular dynamics simulations (also known as discrete element $\operatorname{method}^{27}$ in the engineering literature) to create static granular packings which mimic real granular matter. Starting from a dilute gas phase, we solve Newton's equations where the particles interact with a frictionless repulsive contact force, called the Hertzian interaction ${ }^{28}$. The centers of the particles are restricted to a two-dimensional plane; however, the Hertzian repulsive force is that of three-dimensional elastic spheres: $F=\frac{2}{3} E /\left(1-\nu^{2}\right) R^{1 / 2} n^{3 / 2}$, where $E$ and $\nu$ are the Young modulus and Poisson ratio of the particle's material, and $R=R_{1} R_{2} /\left(R_{1}+R_{2}\right)$. The overlap $n=R_{1}+R_{2}-r_{12}$ is calculated from the particle's radii $R_{1}$ and $R_{2}$ and their centers' distance $r_{12}$. In some of the analysis, we used the frictional Hertz-Mindlin 28 force law. During the evolution we shrink the volume of the periodic box with rate controlled by a feedback loop to achieve a target pressure. As we include some dissipation in the particle interactions, eventually all motion stops and a static granular packing is obtained in mechanical equilibrium. For each set of parameters we use 100 independent packings of 10,000 particles in two dimensions, with polydispersity ranging 5\%-20\% (maximum deviation from the mean, using flat distribution of radii). By extracting re- 
cursively subsystems with half the number of grains, in total five system sizes were obtained. The pressures are expressed in units of the Young modulus of the particle's material. For more details see ref. 7 .

Monte Carlo. The Edwards ensemble for a granular heap is a uniform probability measure on the forces subject to equations for the balance of each particle and inequalities for the positivity of each contact force. In an appropriately reduced space of forces this represents a constant non-zero probability density inside a convex polyhedron. We sample this distribution by the following stochastic process. Starting with a point inside the polyhedron we select a line in a random direction (from a discrete set of directions). In this direction we calculate the intersections with the polyhedron. A new point is chosen on the line with uniform probability between the intersection points. Averages calculated from such a process converge to unweighted averages over the whole polyhedron. For more details see ref. 29.

1. Garcia-Rojo, R., Herrmann, H. J. \& McNamara, S. Powders and Grains 2005 (Balkema, 2005).

2. Jaeger, H. M., Nagel, S. R. \& Behringer, R. P. Granular solids, liquids and gases. Rev. Mod. Phys. 68, 1259-1273 (1996).

3. Daerr, A. \& Douady, S. Two types of avalanche behaviour in granular media. Nature 399, 241-243 (1999).

4. Makse, H. A., Gland, N., Johnson, D. L. \& Schwartz, L. M. Why effective medium theory 
fails in granular materials. Phys. Rev. Lett. 83, 5070-5073 (1999).

5. Goldenberg, C. \& Goldhirsch, I. Friction enhances elasticity in granular solids. Nature 435, 188-191 (2005).

6. Jia, X., Caroli, C. \& Velicky, B. Ultrasound propagation in externally stressed granular media. Phys. Rev. Lett. 82, 1863-1866 (1999).

7. Somfai, E., Roux, J.-N., Snoeijer, J. H., van Hecke, M. \& van Saarloos, W. Wave propagation in confined granular systems. Phys. Rev. E 72, 021301 (2005).

8. Vanel, L., Howell, D., Clark, D., Behringer, R. P. \& Clement, E. Memories in sand: Experimental tests of construction history on stress distributions under sandpiles. Phys. Rev. E 60, R5040-R5043 (1999).

9. Wittmer, J. P., Claudin, P., Cates, M. E. \& Bouchaud, J.-P. An explanation for the central stress minimum in sand piles. Nature 382, 336-339 (1996).

10. Geng, J. et al. Footprints in sand: The response of a granular material to local perturbations. Phys. Rev. Lett. 87, 035506 (2001).

11. Reydellet, G. \& Clement, E. Green's function probe of a static granular piling. Phys. Rev. Lett. 86, 3308-3311 (2001).

12. Majmudar, T. S. \& Behringer, R. P. Contact force measurements and stress-induced anisotropy in granular materials. Nature 435, 1079-1082 (2005).

13. Edwards, S. F. \& Oakeshott, R. Theory of powders. Physica A 157, 1080-1090 (1989). 
14. Makse, H. A. \& Kurchan, J. Testing the thermodynamics approach to granular matter with a numerical model of a decisive experiment. Nature 415, 614-617 (2002).

15. Snoeijer, J. H., Vlugt, T. J. H., van Hecke, M. \& van Saarloos, W. Force network ensemble: a new approach to static granular matter. Phys. Rev. Lett. 92, 054302 (2004).

16. Liu, C.-h. et al. Force fluctuations in bead packs. Science 269, 513-515 (1995).

17. Coppersmith, S. N., h. Liu, C., Majumdar, S., Narayan, O. \& Witten, T. A. Model for force fluctuations in bead packs. Phys. Rev. E 53, 4673-4685 (1996).

18. da Silva, M. \& Rajchenbach, J. Stress transmission through a model system of cohesionless elastic grains. Nature 406, 708-710 (2000).

19. Radjai, F., Jean, M., Moreau, J.-J. \& Roux, S. Force distributions in dense two-dimensional granular systems. Phys. Rev. Lett. 77, 274-277 (1996).

20. Makse, H. A., Johnson, D. L. \& Schwartz, L. M. Packing of compressible granular materials. Phys. Rev. Lett. 84, 4160-4163 (2000).

21. Mueth, D. M., Jaeger, H. M. \& Nagel, S. R. Force distribution in a granular medium. Phys. Rev. E 57, 3164-3169 (1998).

22. Blair, D. L., Mueggenburg, N. W., Marshall, A. H., Jaeger, H. M. \& Nagel, S. R. Force distributions in $3 \mathrm{~d}$ granular assemblies: Effects of packing order and inter-particle friction. Phys. Rev. E 63, 041304 (2001). 
23. Erikson, J. M., Mueggenburg, N. W., Jaeger, H. M. \& Nagel, S. R. Force distributions in three-dimensional compressible granular packs. Phys. Rev. E 66, 040301 (2002).

24. Fortuin, C. M. \& Kasteleyn, P. W. On the random cluster model. Physica 57, 536-564 (1971).

25. Stauffer, D. \& Aharony, A. Introduction to Percolation Theory (Taylor \& Francis, 1991).

26. Brujic, J. et al. 3d bulk measurements of the force distribution in a compressed emulsion system. Faraday Disc. 123, 207-220 (2003).

27. Cundall, P. A. \& Strack, O. D. L. A discrete numerical model for granular assemblies. Géotechnique 29, 47-65 (1979).

28. Johnson, K. L. Contact mechanics (Cambridge University Press, Cambridge, 1985).

29. Ostojic, S. \& Panja, D. Response of a hexagonal granular packing under a localized external force. Europhys. Lett. 71, 70-77 (2004).

Acknowledgements We thank B. Behringer, W. Ellenbroek, M. van Hecke, C. Goldenberg, W. van Saarloos and K. Shundyak for discussions. SO is financially supported by the Dutch research organization FOM (Fundamenteel Onderzoek der Materie). ES is supported by the PHYNECS training network of the European Commission.

Competing Interests The authors declare that they have no competing financial interests.

Correspondence Correspondence and requests for materials should be addressed to B.N. (email: nienhuis@science.uva.nl). 

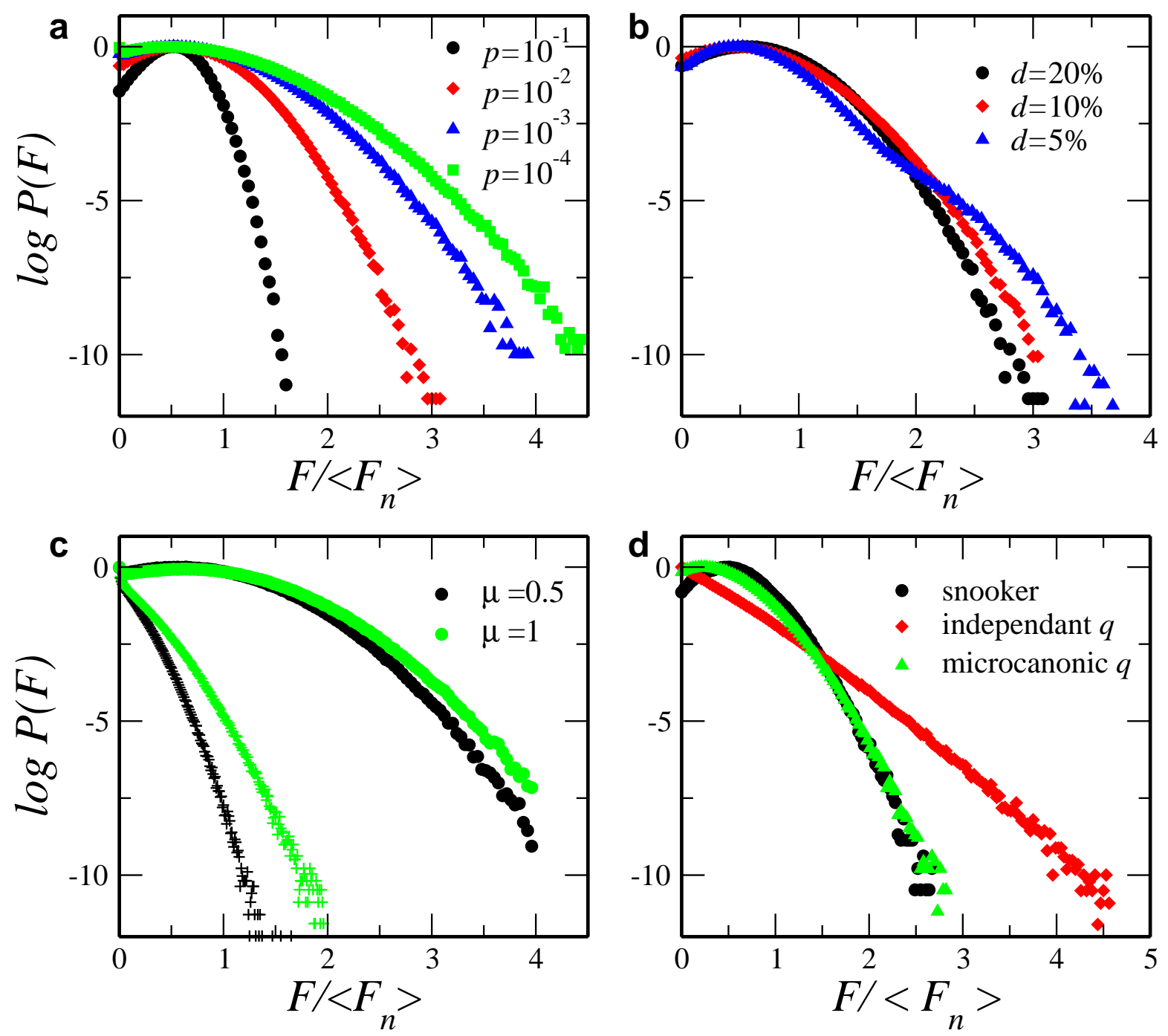

Figure 4: *

Supplementary Figure: Probability distributions $P(F)$ of force magnitudes in all the systems studied. All the forces are rescaled by the mean normal force $<F_{n}>$. (a-c), Dependence of $P(F)$ on the parameters varied in the molecular dynamics simulations: (a) pressure, (b) polydispersity and (c) friction. In (c), the distribution of normal forces is represented by the symbols $\bullet$, and the tangential forces by + . (d), $P(F)$ in the three lattice models mentioned in the text. 